\title{
A look back at 2019 in BioTechniques
}

To all of our readers, we would first like to wish you a happy New Year and introduce you to the 68th volume of BioTechniques.

$\overline{\text { Joseph Martin*,1 \& Tristan Free' }}$

W

e will begin by taking a look back at 2019; what a year it's been! We have continued to grow the journal and improve both author and reader experience. In this short piece we would like to highlight the most downloaded peerreviewed journal content and delve into the impact of our content on multiple platforms using Altmetrics. Additionally, we would like to take a look at our digital content highlights along with what we have in store for 2020 .

\section{JOURNAL CONTENT HIGHLIGHTS}

At the time of writing, the most downloaded BioTechniques article of 2019 was entitled "Triplicate PCR reactions for $16 \mathrm{~S}$ rRNA gene amplicon sequencing are unnecessary" [1]. With over 4600 downloads, this report by Rob Knight and his UCSF team demonstrated that single PCR reactions are sufficient for microbiome analysis.

"We wanted to find out if triplicate PCR was really necessary, or whether it was a myth passed down through generations of grad students from the dawn of amplicon analysis," commented lead author, Rob Knight (University of California San Diego, CA, USA).

This article has definitely made an impact in the field, evident from the 115 tweets, with

\section{${ }^{66}$ Moving into 2020, the reproducibility of our} research outputs will continue to be a priority for us."

'Future Science Group, Unitec House, 2 Albert Place, London, UK; *Author for correspondence: jmartin@ biotechniques.com

BioTechniques 68: 2-3 (January 2020) 10.2144/ btn-2019-0164 an upper bound of 185,000 followers. This highlights the power of social media to reach a wide audience.

Next up, also accumulating over 4600 downloads is the review entitled "Guidelines for optimized gene knockout using CRISPR/ Cas9". In this review, Kruys et al. present guidelines and tools to optimize CRISPR/ Cas9 genome-targeting efficiency and specificity [2]. With the continued evolution of CRISPR/Cas9 technology it is not surprising that this is one of the most popular articles with our audience.

The review entitled "Recent advances in photo-crosslinkable hydrogels for biomedical applications" by Cowie et al. discussed some of the existing challenges and future perspectives of engineering photo-crosslinkable hydrogels for biomedical applications, whilst also delving into the advantages of photopolymerization [3].

We have continued to measure the influence of our publications through more than just the number of downloads. Altmetrics is a powerful way to measure this. It collates information from multiple different sources across different platforms to show the way in which research is being broadcast across the world.

Interestingly, the most prominent example of this comes from a paper that was only published at the end of November. This 'ahead of print' report describes a methodology for the at-home collection of urine samples for prostate cancer detection [4]. As you can see from the 'Altmetric Attention Score' for the research output illustrated in Figure 1, this article has already been reported by nine news outlets, 38 tweets, one blog and one Facebook page, putting the article in the top $5 \%$ of all research outputs ever tracked by Altmetric.

Moving into 2020, the reproducibility of our research outputs will continue to be a priority for us. Protocol sharing will still be encouraged with every publication through Protocols.io [5], a collaborative platform for scientific protocols with the aim to improve the reproducibility of all published papers by providing authors better ways to share methodological details behind their work, in a more dynamic and interactive form. A a recent example is demonstrated by the protocol by Tröscher et al., which presents an approach to enable monitoring gene expression in vivo on a single-cell basis over time [6].

\section{DIGITAL CONTENT HIGHLIGHTS}

Over on the BioTechniques.com website, 2019 has been a transformative year. The launch of a new site, the introduction of our podcast series Talking Techniques and Talking Tech News, and our virtual symposium, Advancing Precision Medicine 2019, are just a few examples of how the digital side of BioTechniques has expanded its avenues to share information.

This expansion has been reflected in our social media following. The BioTechniques Facebook page now has over 650,000 followers - an increase of 600,000 from 2018 - and our twitter page has more than 10,000.

This expanding following has led to our content reaching an increasing number of people and our top content that has featured on the site in 2019 is as follows:

1. Why time flies so fast as we get older [7] 2. Not-So Identical Twins [8]

3. Fermented foods: activating the immune system [9]

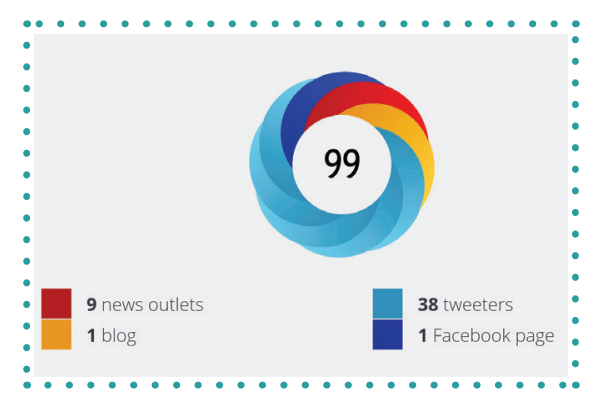

Figure 1. Altmetric attention score for "A

methodology for the at-home collection of urine samples for prostate cancer". 
The most highly viewed content from our new podcast and videos sections also impressive. The Talking Tech News: 'Cerebral organoids: past, present, future', featuring Assistant Editor Jennifer Straiton [10], has been listened to over 2000 times, and our video interview with Pat Brown, CEO of Impossible Foods and inventor of DNA Microarrays [11], has been visited over 1000 times.

Our digital content has been read by over 974,000 digital users from more than 200 countries and states, signalling BioTechniques' ever expanding and diversifying audience as we aim to build on our reputation as a journal and site of global renown and importance.

If you are active on social media, be sure to follow and connect with us on Facebook (@BioTechniques), Twitter (@MyBioTech- niques), Instagram (@mybiotechniques) and LinkedIn (BioTechniques).

\section{CONCLUSION}

We would like to thank our readers, contributing authors, valued Editorial Board members and peer reviewers for their continued support and collaboration as we continue to publish the latest cutting-edge research. We very much look forward to working with you all over the next year and to seeing BioTechniques continue to grow and serve the research community in 2020 .

There are many opportunities to be featured both in the journal and on the website, whether in a written interview or opinion piece, a podcast or video, so do get in touch with Managing Editor Joseph Martin (jmartin@biotechniques.com) to find out more.

\section{REFERENCES}

1. Maritz C, Sharma A, Humphrey G et al. Triplicate PCR reactions for $16 S$ rRNA gene amplicon sequencing are unnecessary. BioTechniques 67(1), 29-32 (2019).

2. Van Campenhout $C$, Cabochette $P$, Veillard $A C$ et al. Guidelines for optimized gene knockout using CRISPR/ Cas9. BioTechniques 66(6), 295-302 (2019).

3. Choi JR, Yong KW, Choi JY, Cowie AC. Recent advances in photo-crosslinkable hydrogels for biomedical applications. BioTechniques 66(1), 40-53 (2019).

4. Webb M, Manley K, Olivan M et al. Methodology for the at-home collection of urine samples for prostate cancer detection. BioTechniques doi:10.2144/btn-2019-0092 (2019).

5. Protocols.io. https://www.protocols.io/

6. Protocol: Spectral recording of gene expression history by fluorescent timer protein. doi:10.17504/protocols. io.8jvhun6 (2019).

7. Why time flies so fast as we get older. https://www. biotechniques.com/cell-and-development-biology/whytime-flies-as-we-get-older/

8. Not-So Identical Twins. https://www.biotechniques. com/omics/not-so-identical-twins/

9. Fermented foods: activating the immune system https://www.biotechniques.com/microbiology-virology/ fermented-foods-activating-the-immune-system/

10. Talking Tech News. Cerebral organoids: past, present future. https://www.biotechniques.com/podcasts/talking-tech-news-cerebral-organoids-past-present-future/

11. Pat Brown: solving humanity's biggest problem. https:// www.biotechniques.com/bioengineering-biophysics/ pat-brown-solving-humanitys-biggest-problem/ 